\title{
Nigerianische OPfer aUS DEM MenSChenhaNdel UND DER ZWANGSPROSTITUTION - FRAGMENTE AUS DER PSYCHOSOZIALEN BERATUNG EINER FACHBERATUNGSSTELLE IN SÜDDEUTSCHLAND
}

\author{
Lena SIEMERS, Ethnologin M.A. / Sozialarbeiterin, München
}

Dieser Beitrag beruht auf den Berichten von nigerianischen Frauen, die Opfer von Menschenhandel und Zwangsprostitution wurden. Im Rahmen psychosozialer Beratung im Jahr 2018 und 2019 lernte ich zahlreiche Betroffene kennen. Dieser Artikel wurde anonymisiert aus den Berichten zweier Klientinnen kompiliert, um die Anonymität der betreffenden Personen unbedingt zu wahren. Er ist aber beispielhaft für die Erlebnisse der Opfer. Die geschilderten Erlebnisse der Frau, die in diesem Bericht Hope genannt wird, sind nicht akkumuliert, sondern wurden durch die Vermischung lediglich verfremdet. Einige der Faktoren, die dazu beitragen, dass Frauen in die Fänge von Menschenhändlern geraten, werden hier angesprochen. Allerdings liefern diese Schilderungen kein umfassendes Bild der Push- und Pull-Faktoren - man könnte an dieser Stelle weiter ausholen und die Ursprünge der Problematik des Menschenhandels historisch zum Beispiel in kolonialen Verbrechen im 15. Jahrhundert suchen. Ich möchte mich aber nur auf die Erzählungen, die mir während meiner Beschäftigung als Sozialarbeiterin zugetragen worden sind, stützen. So beginnt dieser Bericht in der Kindheit von Hope, als in ihrem konkreten Fall der Menschenhandel bereits seinen Anfang nahm.

Hope lebte mit fünf Geschwistern und ihren Eltern in einem kleinen Dorf in der Nähe von Benin City in Edo State in Nigeria. Ihr Überleben sicherte die Familie durch Arbeit auf der Farm eines großen Bauern. Mehr als einen Raum, den sie angemietet hatten, konnte sich die Familie nicht leisten. So schliefen und wohnten alle sieben Familienmitglieder in einem Raum. 
Nigeria ist das bevölkerungsreichste Land Afrikas und steht an siebter Stelle der bevölkerungsreichsten Länder der Erde. Alleine Lagos hat beinahe so viele Einwohner wie ganz Ghana. ${ }^{1}$ Verschiedenen Schätzungen zufolge leben mehr als 70 Prozent der Bevölkerung Nigerias in Armut, während eine extrem reiche Oberschicht vom Öl- und Gashandel profitiert. Nach der „World Poverty Clock“, die sich aus Daten des „World Data Lab“ speist, leben in Nigeria (Stand Dezember 2019) 47,7 Prozent der Bevölkerung in extremster Armut und Prognosen besagen, dass sich dieser Anteil noch erhöhen wird. Extreme Armut ist definiert als der Zustand, dass eine Person weniger als 1,90 \$ pro Tag zum Leben zur Verfügung hat (CRESPO CUARESMA et al. 2018). In Nigeria leben weltweit die meisten von extremer Armut betroffenen Menschen - eine traurige Spitzenposition! ${ }^{2}$ Geflüchtete aus Nigeria werden in Europa allerdings oft als ,Wirtschaftsflüchtlinge" bezeichnet, was in Anbetracht des Ausmaßes der Armut in diesem Land wie ein Euphemismus erscheint. Verwunderlich ist auch, dass der deutsche Bundestag Schriftstücke herausgibt, in welchen propagiert wird, dass Nigerianer/innen keinen Grund hätten, zur Flucht aufzubrechen, da „(...) dort das Pro-Kopf-Einkommen höher ist als in vielen Ländern Afrikas. “3 So kann man von offizieller Seite die Armutssituation eines Landes schönreden, indem man die Spitzeneinkommen der extrem reichen Oberschicht, die ihren Profit aus Öl und Gas schlägt, einfach auf die ganze Bevölkerung umrechnet, die in Wahrheit zum größten Teil in extremer Armut lebt. Diese Propaganda ist ein gefundenes „Fressen“ für viele Medien und so nimmt die Diskriminierung nigerianischer Geflüchteter ihren eigenen Lauf. Für den Großteil der Bevölkerung Nigerias bedeutet extreme Armut: keine bezahlbare Krankenversorgung, keinen Zugang zu sauberem Wasser und mangelnde Schulbildung. ${ }^{4}$ Da die Eltern das Schulgeld nicht aufbringen können, werden oft nicht alle Kinder zur Schule geschickt, oder sie können die Schule nur unregelmäßig besuchen. Selbst das Absolvieren einer öffentlichen Primary und Secondary School garantiert nicht, dass man danach flüssig lesen und schreiben kann. Die Folgen dieser extremen Armut sind verheerend: In der Hoffnung, den Kindern ein einigermaßen gutes Leben ermöglichen zu können, werden sie oft zu entfernten Verwandten geschickt, im Notfall aber auch verkauft oder verstoßen. Das führt wiederum zu Kinderarbeit und häufig auch zu sexuellem Missbrauch. Sollten die Eltern oder nur ein Elternteil gar verstorben sein, sind die Auswirkungen für die Kinder noch gravierender, da ihnen keinerlei Schutz mehr geboten wird.

Hope sei sich schon als Kind sicher gewesen, dass mit ihr etwas nicht stimme. Die Kindheitserinnerungen von Hope fokussieren sich auf die Erinnerung, dass - wenn sie sich alleine in einem Raum aufhielt und ihre Beine übereinanderschlug - sie von einem fremden Geist besessen worden sei. Sie berichtet, dass sie dann Stimmen hörte und sich fremdbestimmt gefühlt habe. Außerdem sei sie gegenüber ihren Eltern rebellisch gewe-

Vgl. <https://www.worldometers.info/world-population/nigeria-population/>.

Vgl. <https://www.ohchr.org/EN/Countries/AfricaRegion/Pages/NGSummary2019.aspx>.

Vgl. <https://www.bundestag.de/resource/blob/550530/c1cb08a51eb3266671698235d55e4b 75/A-Drs-19-4-26-I-neu-data.pdf $>$.

$4 \mathrm{Vgl} .<\mathrm{https}: / / \mathrm{www}-\mathrm{cdn}$. oxfam.org/s3fs-public/file_attachments/cr-inequality-in-nigeria-170 517-en.pdf>. 
sen und schnell aggressiv geworden. Hopes Mutter besuchte regelmäßig eine Pfingstkirche im Dorf und bemühte sich immer darum, dass ihre Kinder sie dorthin begleiteten. Hope verweigerte den regelmäßigen Kirchenbesuch aber vehement. Rückschauend sagt sie heute, dass sie Angst gehabt hätte, der Pfarrer würde erkennen, dass etwas mit ihr nicht stimmt und dass er dies vor der Familie und den anderen Kirchenbesucher/inne/n laut ausgesprochen hätte, was für sie schlimme Konsequenzen gehabt hätte.

In Nigeria existiert das Phänomen spiritueller Stigmatisierungen, die dazu führen, dass Kinder verstoßen, missbraucht und ausgebeutet werden. Beispielhaft zu nennen sind hier Epilepsie und Sichelzellenanämie, beides Krankheiten, die von Krampfanfällen begleitet sind. Besonders in ländlichen Regionen, wo es sowieso fast keine Gesundheitsversorgung gibt, werden diese Krampfanfälle häufig als spirituelles Phänomen identifiziert. Ohne beurteilen zu wollen und zu können, inwieweit Krampfanfälle auf einer spirituellen Ebene bei einem gesunden Menschen ausgelöst werden können, kann man dennoch feststellen, dass auf jeden Fall einige kranke (physisch und psychisch) Menschen von dieser Stigmatisierung betroffen sind. Inzwischen untersuchen Studien den Zusammenhang zwischen Sichelzellenanämie und der Stigmatisierung als Ogbanje (s.u.). Für Hope kommen diese Erkenntnisse aber leider zu spät (NzEwı 2001).

Hopes Mutter schaffte es, als Hope elf Jahre alt war, eines Tages dennoch sie in die örtliche Kirche namens „Mountain of Fire“5 zu einem „Deliverance Program“ mitzunehmen. Es handelt sich bei „Mountain of Fire“ um eine pfingstkirchliche Bewegung, die für ihre „Teufelsaustreibungen“ bekannt ist.

Als Hope dann zum Pastor nach vorne gerufen wurde, hat er ihr vor der versammelten Gemeinde gesagt, dass sie eine Ogbanje sei. Als Ogbanje werden Kinder bezeichnet, von denen man glaubt, sie würden sterben und immer wieder von der gleichen Mutter wiedergeboren werden. Sie kommen aus der Welt der Wassergeister und stehen in direktem Kontakt mit diesen. Sollten Ogbanjes nicht sterben, dann wird ihnen nachgesagt, dass sie abnorme psychische Verhaltensweisen zeigen und in Kontakt mit Wassergöttern stehen, die von der nigerianischen Gesellschaft sehr gefürchtet werden (IleChUKwU 2007). Wenn sie der Familie keinen Reichtum bringen, dann sind sie dazu da der Familie Unglück zu bringen. Auf der offiziellen Website der „Mountain of Fire“Kirche steht im Download-Bereich ein Dokument mit Gebeten zur Verfügung, die wohl im Rahmen eines Live Events am 16.12. 2015 veröffentlicht wurden. Das Thema der Gebete lautet „Set your Environment Free“. Neben Gebeten zur Befreiung von satanischen Machenschaften und Homosexualität in der Nachbarschaft findet man unter „Section 3“ auch ein Gebet zum Schutz vor den gefürchteten Wassergeistern: „Every marine altar in my environment, catch fire, in the name of Jesus. " 6

Es ist nicht weiter verwunderlich, dass Hope sich weigerte, dem Pastor gegenüber einer Verbindung zu Wassergeistern zuzugeben und zu „beichten“, dass sie eine

5 Es handelt sich bei „Mountain of Fire“ um eine pfingstkirchliche Bewegung, die für ihre Teufelsaustreibungen bekannt ist; siehe auch: <http://www.mountainoffire.org/about $>$.

$6 \quad \mathrm{Vgl} .<\mathrm{cdn}$.mountainoffire.org/docs/specials/121615-wed-service-environmental-prayers.pdf $>$. 
Ogbanje ist. Denn sie wusste, wenn sie das zugibt, dann wird der Pastor eine Austreibung der Wassergeister bei ihr vornehmen und davor hatte sie große Angst.

Nach dem Besuch in der Kirche und ihrer Weigerung, dem Pastor gegenüber ihr „Problem“ zuzugeben, begann ihre Mutter Hope regelmäßig zu schlagen. Da die Familie nicht mit Reichtum gesegnet war, suchte die Mutter die Schuld dieses Zustandes vermutlich in Hopes „spirituellem Problem.“ Da der größte Teil der nigerianischen Bevölkerung unter extremer Armut leidet, führt dies unter anderem zu extremen Gewalttaten, die eine sozioökonomische Abwärtsspirale in Gang halten.

So klopfte es eines Tages an der Tür des Zimmers der Familie und vier fremde Männer standen davor. Mit vorgehaltenen Waffen forderten sie den Vater von Hope auf, ihnen Geld zu geben. Der Vater hatte kein Geld zuhause und sagte dies den Männern auch, während Hope, ihr kleiner Bruder und ihre ältere Schwester Destiny hinter ihm standen. Als die Männer realisierten, dass der Vater ihnen kein Geld geben konnte und würde, drangen sie in den Raum ein und versuchten Hopes Schwester Destiny festzuhalten und zu vergewaltigen. Destiny wehrte sich aber mit allen Kräften, woraufhin die Männer sie einfach erschossen haben. Dann schnappten sie sich Hope und vergewaltigten sie mehrmals vor den Augen ihres Bruders und ihres Vaters, während die Leiche ihrer Schwester auf dem Boden lag. Die Familie konnte ihr nicht helfen, da die Waffen der Männer immer noch auf sie gerichtet waren und sie bereits gelähmt waren vor Angst. Die Männer verließen danach das Haus und liefen davon. Hope wurde von dieser Vergewaltigung schwanger und gebar noch im gleichen Jahr, da war sie 14 Jahre alt, einen Sohn. Die Familie von Hope schämte sich gegenüber ihren Nachbarn sehr für diesen Vorfall und die daraus resultierende Schwangerschaft.

In Nigeria werden Opfer sexualisierter Gewalt und die Kinder, die aus solchen Übergriffen entstehen, enorm stigmatisiert. Die Familien der Opfer fühlen sich durch die Stigmatisierung so unter Druck gesetzt, dass sie versuchen, die Geschehnisse zu verheimlichen (ACHUNIKE \& KiTAuSE 2014). Vergewaltigung scheint in Nigeria ein weit verbreitetes Problem zu sein, zuverlässige Zahlen gibt es dazu nicht, da die meisten Fälle erst gar nicht zur Anzeige gebracht werden. Bei den von mir in der Fachberatungsstelle betreuten Klientinnen hat der Großteil aber in der Kindheit oder als Jugendliche sexualisierte Gewalt erlebt.

Hopes Mutter kümmerte sich um den Sohn, er wurde Hope anfangs nur zum Stillen angelegt. Eine Nachbarin erfuhr von dem Vorfall und unterbreitete den Vorschlag, dass Hope nach Europa zu ihrer Tochter gehen könne, um sich dort um deren Haushalt zu kümmern. Glaubhaft hat diese Frau versichert, dass dies Hopes große Chance sei. Für Hopes Familie war dies eine willkommene Lösung, die problematische Tochter loszuwerden und noch etwas Hoffnung auf Geldsendungen aus Europa haben zu können. Der Vater von Hope stimmte letztendlich diesem Vorschlag zu. Die Tochter der Nachbar/ inne/n erklärte sich bereit, die „Reisekosten“ für Hope vorzustrecken. Um das Arrangement vertraglich festzuhalten, brachten beide Familien Hope zu einem Juju-Schrein nicht sehr weit vom Wohnort entfernt. 
„Juju“ ist ein umgangssprachlicher Überbegriff für Praktiken „traditioneller“ Religionen Westafrikas. Die einzelnen Religionen haben meist keinen Namen und werden, auch durch den vermehrten Einfluss christlicher Kirchen, gerne unter dem Überbegriff „Juju“ zusammengefasst, in ihrer Ausprägung sind sie aber je nach Region und Kontext unterschiedlich.

In einem Juju-Schrein wurde Hope von beiden Familien dazu gezwungen, einen Schwur abzulegen. Zu diesem Zweck wurde ein Huhn geschlachtet und Hope musste dessen Blut trinken. Sie musste schwören, dass sie niemandem von ihrer Reise erzählt, nicht zur Polizei geht und die Kosten für die Reise zurückzahlt. Hope erinnert sich, dass es sich um einen Schrein handelte, der mit den Gottheiten Olokun und Eshango arbeitete. Bei Eshango handelt es sich um die Gottheit des Donners und bei Olokun um den Sohn des Schöpfergottes Osanobua. Olokun ist der Herrscher über das Wasser und für Wohlstand und Reichtum zuständig. Hope wurden mit einem Messer zudem viele Schnitte am Körper zugefügt, die bis heute als Narben sichtbar sind.

Tiefe, senkrechte Schnitte zwischen den Brüsten, auf dem Rücken, an den Knien und an den Füßen sollten sichtbar vernarben. Die Wunden bluteten sehr stark und in die offenen Schnitte drückte der Priester eine schwarze Paste, die sehr brannte. Aufgrund dieser schwarzen Paste sind die Schnitte für immer in der Haut sichtbar. Im Gespräch zeigte mir Hope alle Narben, es handelt sich um jeweils in unterschiedlicher Anzahl vertikal angebrachte Schnitte. Die jeweilige Anzahl der Schnitte steht für die verschiedenen Versprechungen, für deren Einhaltung ein Vertrag mit jeweils einer Gottheit abgeschlossen wurde (vgl. Abbildung 1).

Während des Besuches im Schrein sagte der Juju-Priester vor beiden Familien, dass Hope eine Ogbanje sei. Auf seine Empfehlung hin kontaktierten Hopes Eltern in den Tagen nach dem Besuch des Schreins und der Bezichtigung durch den Priester eine weibliche traditionelle Priesterin. Mit ihr und Hope gingen sie gemeinsam zum örtlichen Ikpoba Fluss, um mit Hilfe der Priesterin Hope vom Ogbanje-Dasein zu befreien. Dort band man ihr ein Seil um die Taille und die Priesterin sollte mit ihr im Fluss baden. Die Eltern erklärten Hope, dass dies dazu diene, Hope den Geist der Ogbanje aus dem Körper zu treiben. Die Priesterin lief allerdings davon und es kam zu keiner Austreibung. Die Eltern suchten die Schuld für die missglückte Austreibung bei Hope, da sie davon ausgegangen waren, dass die Priesterin zu große Angst vor Hope hatte. In den Tagen danach wurde Hope wiederholt von ihrer Mutter geschlagen, dieses Mal aber heftiger als die Jahre zuvor. Vor der Abreise gaben die Eltern Hope eine blaurote Glasperlenkette, die sie sich um die Taille binden musste.

2006 begab sich Hope dann auf die Reise. Zunächst mit dem Bus nach Agadez/ Niger und weiter auf offenen LKWs nach Tripolis. Die Reise war sehr anstrengend und obwohl sie alleine mit einer großen Gruppe fremder Menschen unterwegs war, passierte ihr weiter nichts. In Libyen wurde Hope vom Schlepper an ein „Connection House“ 7 verkauft.

7 In einem Connection House werden in Libyen Geflüchtete meist unfreiwillig eingesperrt und zur Prostitution gezwungen. 
Abb. 1: Körpermarkierungen, die Hope im Juju-Schrein im Rahmen des Schwures zugefügt wurden.
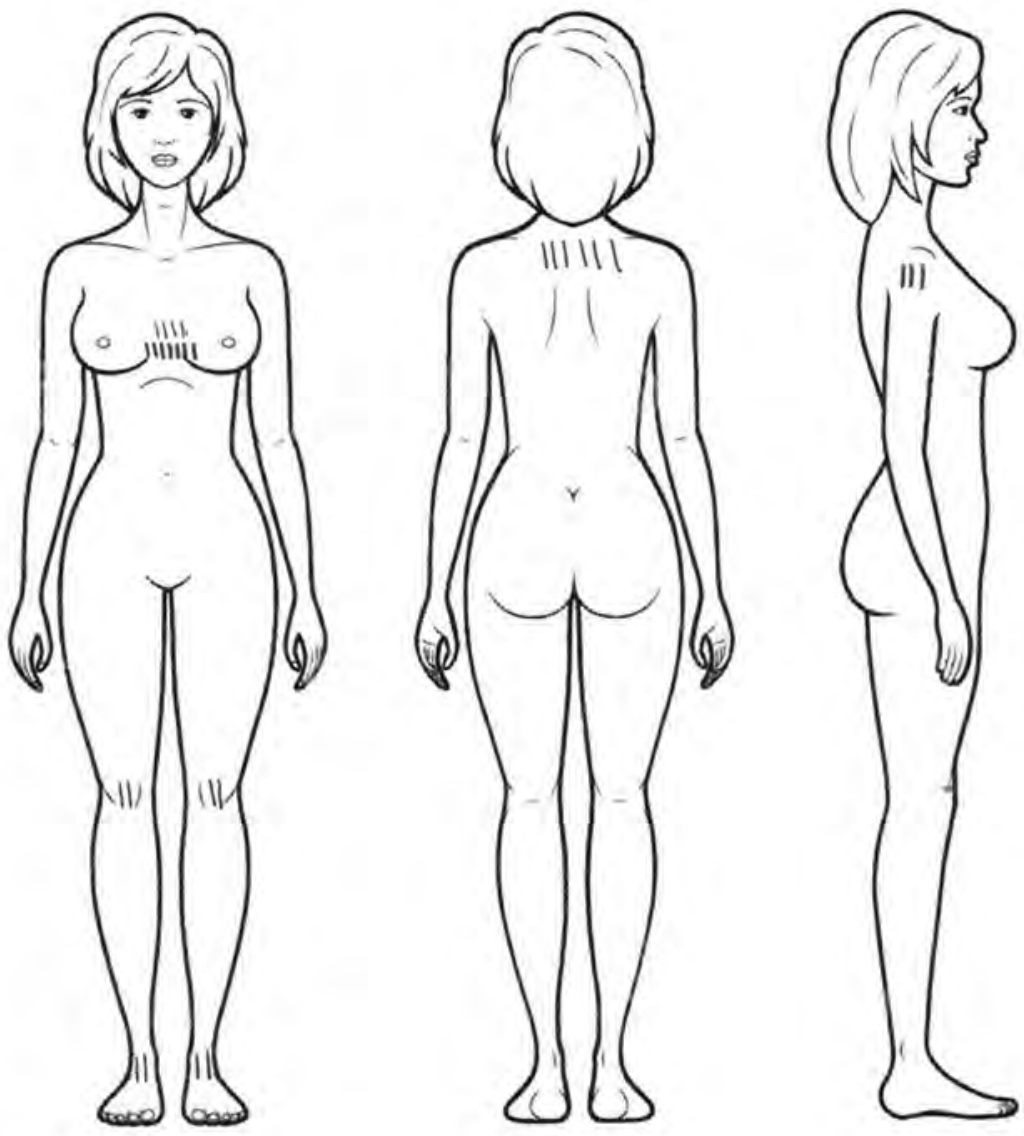

Amnesty International berichtet regelmäßig, dass Geflüchtete in Libyen Opfer schwerer und systematischer Menschenrechtsverletzungen und -verstöße werden. Täter sind Mitarbeiter von Hafteinrichtungen und der Häfen, Schleuser und bewaffnete Gruppen. ${ }^{8}$ Den Schilderungen meiner Klientinnen zufolge kann man davon ausgehen, dass Nigerianer/innen (im Besonderen die Frauen) in Libyen als vogelfrei gelten. Sich alleine auf der Straße zu bewegen ist zu gefährlich, bei Gefahr kann man keine Hilfe von der Polizei erwarten und bewaffnete Gruppen ziehen durch die Straßen, auf der Suche nach schutzlosen Menschen, die sie einfangen, ausrauben, misshandeln und weiterverkau-

$8 \quad$ Vgl. <https://www.amnesty.de/jahresbericht/2018/libyen>. 
fen können. Oft wird berichtet, dass solche Gruppen sich Asma Boys nennen und aus uniformierten jungen Libyern bestehen. ${ }^{9}$ Einige meiner Klientinnen berichteten davon, dass sie von solchen Asma Boys entführt wurden, teilweise vergewaltigt und dann an ein sogenanntes Connection House verkauft wurden.

Beim Eintreffen in einem Connection House wird dem Mädchen oft vorgerechnet, wie viel der/die Besitzer/in des Connection Houses für das Mädchen gezahlt hat und wieviel Dinar sie wieder abbezahlen müsse, um wieder freizukommen. Im Fall von Hope waren es 800 Dinar, die sie dem Besitzer, nach dessen Vorstellung, schuldete, da er sie angeblich für 400 Dinar gekauft hatte. Insgesamt waren dort etwa zehn Mädchen. Die anderen Mädchen gaben Hope Kleidung, Hygiene-Artikel (Seife, Parfum) und eine Packung Kondome. Ihr wurde gesagt, dass sie das Geld für diese Artikel zurückzahlen müsste, was sie auch per Unterschrift zu bestätigen hatte. Eine Frau im Connection House sagte zu ihr, sie sollte froh sein, dass sie nun in Sicherheit sei. Hope erwiderte jedoch, dass sie sich hier nicht sicher fühlte. Die Frau forderte sie auf, ihren Mund zu halten, da sie ansonsten „Araber“ anrufen würde, die sie in die Wüste bringen und dort töten würden. Hope hatte also keine andere Wahl, als sich zu prostituieren. Die Mädchen waren alle zusammen in einem großen Raum untergebracht, nur durch Leintücher waren die Bereiche der einzelnen Bewohnerinnen voneinander abgetrennt. Den Mädchen wurden Sätze auf Arabisch beigebracht, damit sie sich mit den Freiern verständigen konnten. Wenn sie sich weigerten, mit den Männern zu schlafen, dann teilten die Freier dies dem Besitzer des Connection House mit und dieser bestrafte die Mädchen dafür. Es kam auch vor, dass die Mädchen weiterverkauft wurden und der offene Geldbetrag wieder von neuem abbezahlt werden musste. Hope bekam 10 Dinar pro Freier, wenn die Freier sie gut fanden, gaben sie ihr manchmal 15 Dinar. Wenn die Freier die Nacht mit den Frauen verbrachten, dann zahlten sie 30 Dinar. Es kam auch vor, dass Mädchen eine ganze Woche bei Freiern bleiben mussten. Der Besitzer des Connection Houses erlaubte dies aber nur, wenn er die Männer kannte. Manchmal wurden die Mädchen entführt, deshalb war er vorsichtig. Wenn der Besitzer das betreffende Mädchen mochte, versuchte er, sie wieder zurückzukaufen. Manchmal musste Hope daher auch mit dem Besitzer schlafen:

„They buy girls, like they are buying clothes. They are pricing people based on how they look." 10

Die Mädchen mussten notieren, wieviel Geld sie verdient hatten und wie viele Freier sie jeweils hatten. Wenn die Mädchen Kondome oder Hygieneartikel benötigten, dann wurde ihnen dies extra berechnet. Wenn sie etwas „falsch“ machten, dann mussten sie Strafe bezahlen, die aufgeschrieben und zu ihren „Schulden“ hinzugefügt wurde. Wenn sie krank wurden, dann waren ebenfalls Extrazahlungen fällig. Eine Abtreibung

$9 \quad$ Vgl. <https://www.zeit.de/2017/18/zwangsarbeit-libyen-fluechtlinge-sklaven>.

10 Zitat der Klientin während des Gesprächs im Büro der Beratungsstelle. 
kostete 400 Dinar. Infolge dieses Systems der „Extrazahlungen“ war es eigentlich unmöglich, die 800 Dinar jemals zurückzuzahlen.

Eines Tages rief die Tochter der Nachbarsfamilie aus Europa an und rechnete mit dem Besitzer des Connection Houses aus, wie viel Geld Hope bereits abbezahlt hatte. Die „Madame“ " ${ }^{11}$ zahlte dann den Rest und Hope war „frei“, um in ein Schlauchboot zu steigen und in Richtung Italien aufzubrechen. Die Überfahrt hatte die Madame für sie organisiert und sie gab ihr telefonische Anweisungen, wie Hope auf das Boot gelangen sollte.

In der Nacht fuhren sie in einem Schlauchboot los, wie man sie aus Reportagen im Fernsehen kennt. Während der ganzen Dauer der Überfahrt schrie Hope vor Angst lauthals. Sie wurden dann aber im Morgengrauen von einem Rettungsschiff aufgenommen und nach Sizilien gebracht. Dort wurde sie in ein Flüchtlingscamp gebracht und mit einem geborgten Telefon rief Hope zunächst ihre Mutter in Nigeria an. Seit sie Nigeria verlassen hatte, hatte Hope keinen Kontakt mehr zu ihrer Familie. Die Mutter freute sich sehr über den Anruf von Hope. Danach rief Hope die Madame an, die ihr sagte, dass sie in Sizilien abgeholt werden würde. Sie sagte ihr, dass sie sich unauffällig aus dem Camp entfernen sollte. Auf der Zufahrtsstraße traf sie dann einen Mann, der sie auf das italienische Festland brachte, wo die Madame auf sie wartete. Allerdings blieben sie nicht lange in Italien, sondern sie fuhren direkt mit dem Zug weiter nach Frankreich.

Anscheinend brachte die Madame Hope ins Asylverfahren und erhielt für sie mittels falscher Angaben bezüglich Alter und Herkunft einen Aufenthaltstitel. Hope weiß nicht, welche Asylgründe für sie von der Madame geltend gemacht wurden, aber sie glaubt, dass es FGM war. Sie weiß auch nicht, welche Art von Aufenthaltstitel ihr gewährt wurde, denn sie kümmerte sich nicht darum und musste lediglich alle drei Monate ihren Aufenthalt verlängern.

Die Madame brachte Hope in einer großen Stadt in Frankreich in einer Wohnung unter. Dort lebte sie mit drei anderen nigerianischen Frauen zusammen, die allerdings älter als sie waren. Auch diese Frauen mussten für die Madame auf dem Straßenstrich arbeiten. Die Frauen kümmerten sich um Hope, aber dennoch musste sie drei Jahre für die Madame in der Nähe eines Bahnhofs als Prostituierte tätig sein. Die Madame kam einmal im Monat und holte das verdiente Geld ab. Sie erzählte Hope, dass sie bei ihr $50000 €$ Schulden hätte, doch Hope konnte sich diesen Betrag gar nicht vorstellen. Im Regelfall bediente sie neun bis zehn Männer täglich für jeweils $20 €$. Zu diesem Zeitpunkt war sie gerade 16 Jahre alt gewesen. Die anderen Frauen drängten sie dazu wegzulaufen, weil sie zu jung für dieses „Gewerbe“ sei. Sie musste auch immer wieder Pausen einlegen, da sie große Schmerzen hatte. Im Regelfall konnte sie der Madame im Monat 1500 bis $1700 €$ bezahlen. Die Madame war damit nie zufrieden und schlug sie oft.

11 Weiterführende Literatur zur Organisation des Menschenhandels z.B.: Kreutzer \& MiLBORN 2008. 
Eines Tages hatte Hope plötzlich sehr starke Bauchschmerzen und begab sich nachmittags in ein Krankenhaus. Nach einigen Untersuchungen teilte man ihr die Diagnose Blinddarmentzündung mit und daraufhin wurde sie operiert. Danach blieb Hope eine Woche im Krankenhaus. Nachdem sie wieder in die Wohnung zurückgekehrt war, rief die Madame an und fragte, wo sie gewesen sei. Sie glaubte Hope zunächst nicht, dass sie wirklich im Krankenhaus war und kam deshalb zu Besuch, befragte auch die anderen Frauen in der Wohnung und ließ sich von Hope die Operationswunden zeigen.

Die Madame glaubte ihr dann und sagte ihr, dass sie nur noch eine Woche zuhause bleiben dürfe und danach wieder arbeiten sollte. Obwohl Hope ihr mitteilte, dass sie immer noch unter starken Schmerzen litt und nicht arbeiten könnte, drohte ihr die Madame schlimme Konsequenzen an, sollte sie nicht wieder nach einer Woche ihrem Erwerb nachgehen. So ging Hope mit starken Schmerzen wieder auf den Strich, nahm aber keine Freier an, da die Schmerzen zu stark waren. Da Hope dann beim nächsten Besuch der Madame kein Geld vorweisen konnte, schlug die Madame sie brutal zusammen. Durch die Schläge erlitt Hope eine große Narbe am Oberschenkel. Außerdem führte die Madame Hope Chilipulver in die Augen und in die Vagina ein. [Anm.: Hope weinte beim Erzählen dieser Details heftig und zitterte am ganzen Körper. Sie zeigte mir überdies die Narbe am linken Oberschenkel.]

Zwei Jahre später hatte Hope den Mut, eines Tages einfach nicht mehr in die Wohnung zurückzukehren. Sie reiste in eine andere Stadt und hatte großes Glück, auf andere, ältere nigerianische Aussteigerinnen zu treffen, die ihr zunächst Arbeit als Hausmädchen verschafften. All die Jahre in Frankreich litt Hope unter dem einmal geleisteten Juju-Schwur. Wenn sie Kontakt zu ihren Familienangehörigen aufnahm, erzählten diese stets, dass die Nachbarin immer nach ihr fragen würden, da Hope von den $50000 €$ bislang lediglich $10000 €$ abbezahlt habe. Hope lebte daher in ständiger Angst, von der Madame wiedergefunden zu werden. Der Juju-Schwur hielt sie immer in seinem Bann und sie litt deshalb unter Schlaflosigkeit und Kopfschmerzen. Aus diesem Grund beschloss sie schließlich nach Deutschland zu gehen, in der Hoffnung, sich dort ein besseres Leben aufbauen zu können. Auf dem Parkplatz eines Bahnhofes fand sie eine Mitfahrgelegenheit per „Blabla Car“ nach Deutschland, wo sie um Asyl ansuchte. Da ihre Familie von der Nachbarsfamilie im Dorf weiterhin bedroht wurde, zog die Familie innerhalb des Dorfes um. Einen Umzug in einen anderen Ort kann sich die Familie aber nicht leisten.

Hope berichtet von Schlafstörungen, Alpträumen, ständigen Kopfschmerzen, schmerzenden Narben vom Juju-Ritual und Jucken am ganzen Körper, nachdem sie über ihre Erlebnisse spricht. Ihre Alpträume haben die Vergewaltigung und den Tod der Schwester in Nigeria und die Gewalterfahrungen durch die Madame zum Thema. Zudem wacht sie des Öfteren nachts mit dem als real empfundenen Gefühl auf, jemand habe sie vergewaltigt. 
Im Ablehnungsbescheid des Bundesamtes für Migration und Flüchtlinge steht, Hope könne nach Nigeria zurückkehren, da sie dort ,aufgrund familiärer Bande Unterstützung zur Sicherung des Existenzminimums“ habe. Mit der „familiären Bande“ meint das Bundesamt die Eltern von Hope, die sie bewusst und freiwillig dem Menschenhandel ausgesetzt haben, da sie für ihre in Schande gefallene Tochter keine andere Zukunft mehr gesehen hatten. Hope kämpft weiterhin zusammen mit einem Anwalt an ihrer Seite um einen legalen Aufenthalt in Deutschland, ist Mutter einer gesunden Tochter geworden und befindet sich in traumatherapeutischer Behandlung. 


\section{Bibliographie}

Achunike H.C. \& Kitause, R.H. (2014), Rape epidemic in Nigeria: cases, causes, consequences and response to the pandemic, International Journal of Research in Applied and Social Sciences, 2 (1), pp. 31-44.

Crespo Cuaresma J., Fengler W., Kharas H. et al. (eds.) (2018), Will the Sustainable Development Goals be fulfilled? Assessing present and future global poverty. Palgrave Communications, 4 (29).

ILECHUKWU S. (2007), Ogbanje/abiku and cultural conceptualizations of psychopathology in Nigeria, Mental Health, Religion \& Culture, 10 (3), pp. 239-255.

Kreutzer M. \& Milborn C. (2008), Ware Frau. Auf den Spuren moderner Sklaverei von Afrika nach Europa.

Nzewi E. (2001), Malovent Ogbanje: Recurrent reincarnation or sickle cell disease? Social Science \& Medicine, 52, pp. 1403-1416.

\section{Internetquellen}

“About MFM” (2015), <http://www.mountainoffire.org/about> [Last Access 22/12/2019].

“Amnesty Report Libyen 2017/18” (2018), <https://www.amnesty.de/jahresbericht/2018/libyen> [Last Access 22/12/2019]

"Inequality in Nigeria, Exploring the drivers" (2017), <https://www-cdn.oxfam.org/s3fs-public/ file_attachments/cr-inequality-in-nigeria-170517-en.pdf $>$ [Last Access 22/12/2019].

"Mountain of Fire Miracles Ministry. Prayer Points. Set your Environment free” (2015), <cdn. mountainoffire.org/docs/specials/121615-wed-service-environmental-prayers.pdf $>$ [Last Access 30/12/2019].

"Nigeria Population (Live)", <https://www.worldometers.info/world-population/nigeria-popu lation/> [Last Access 22/12/2019].

“ONHCR in Nigeria“"(2019), <https://www.ohchr.org/EN/Countries/AfricaRegion/Pages/NGSum mary2019.aspx $>$ [Last Access 22/12/2019].

"Sie sind Sklaven" (2017), <https://www.zeit.de/2017/18/zwangsarbeit-libyen-fluechtlingesklaven> [Last Access 22/12/2019].

„Stellungnahme Anhörung des Innenausschusses des Deutschen Bundestages 16. April 2018, Gerald Knaus, Europäische Stabilitätsinitiative (ESI)“ (2018), <https://www.bundestag.de/ resource/blob/550530/c1cb08a51eb3266671698235d55e4b75/A-Drs-19-4-26-I-neu-data. pdf $>$ [Last Access 22/12/2019]. 